\title{
OSCILLATORY BEHAVIOR OF A CERTAIN CLASS OF SECOND-ORDER NONLINEAR PERTURBED DYNAMIC EQUATIONS ON TIME SCALES
}

\author{
SAMIR H. SAKER
}

\begin{abstract}
This paper is concerned with the asymptotic behavior of solutions of the second-order nonlinear perturbed dynamic equation

$$
\left.\left(r(t) x^{\Delta}(t)\right)^{\Delta}+F\left(t, x^{\sigma}\right)\right)=G\left(t, x^{\sigma},\left(x^{\Delta}\right)^{\sigma}\right)
$$

on a time scale $\mathbb{T}$. By using a new technique we establish some sufficient conditions which ensure that every solution oscillates or converges to zero. Our results improve the known oscillation results on the literature for the perturbed dynamic equations on time scales. Some examples illustrating our main results are given.
\end{abstract}

\section{Introduction}

The theory of time scales, which has recently received a lot of attention, was introduced by Stefan Hilger in 1988 in his Ph. D. Thesis in order to unify continuous and discrete analysis (see [18]). The theory of dynamic equations unifies the theories of differential equations and difference equations and it also extends these classical cases to cases "in between", e.g., to so called $q$-difference equations. Since then several authors have expounded on various aspects of this new theory, we refer to the survey paper by Agarwal, Bohner, O'Regan, and Peterson [1] and the references cited therein. A book on the subject of time scales, by Bohner and Peterson [6], summarizes and organizes much of time scale calculus, see also the book by Bohner and Peterson [7] for advances in dynamic equations on time scales.

In recent years there has been much research activity concerning the oscillation and nonoscillation of solutions of dynamic equations on time scales, we refer the reader to the papers [2]-[5], [8]-[16], [19]-[23].

Received June 24, 2005.

2000 Mathematics Subject Classification. 34K11, 39A10, 39A99, 34A99, 34C10, 39A11.

Key words and phrases. oscillation, perturbed dynamic equations, time scale. 
For oscillation of perturbed dynamic equations on time scale Bohner and Saker [9] considered the equation

$$
\left(r(t)\left(x^{\Delta}\right)^{\gamma}\right)^{\Delta}+F\left(t, x^{\sigma}\right)=G\left(t, x^{\sigma},\left(x^{\Delta}\right)^{\sigma}\right) \quad \text { for } t \in\left[t_{0}, \infty\right)_{\mathbb{T}},
$$

on a time scale $\mathbb{T}$, where $\gamma$ is a positive odd integer and

$$
\int_{t_{0}}^{\infty}\left(\frac{1}{r(t)}\right)^{\frac{1}{\gamma}} \Delta t=\infty .
$$

The authors in [9] used the substitution

$$
w(t):=\frac{z^{2} p x^{\Delta}}{x}(t)
$$

and established some sufficient conditions in terms of the coefficients and the graininess function which guarantee that every solution is oscillatory. In this paper we will use a new substitution to establish some new sufficient conditions for oscillation of the second-order nonlinear perturbed dynamic equation

$$
\left(r(t) x^{\Delta}(t)\right)^{\Delta}+F\left(t, x^{\sigma}\right)=G\left(t, x^{\sigma}(t),\left(x^{\Delta}\right)^{\sigma}\right) \text { for } t \in\left[t_{0}, \infty\right)_{\mathbb{T}},
$$

on a time scale $\mathbb{T}$, where $\sigma(t)$ is the forward jump operator. The equation will be studied under the following assumptions:

$\left(A_{1}\right) r: \mathbb{T} \rightarrow \mathbb{R}^{+}$is a positive and $r d$-continuous function;

$\left(A_{2}\right) F: \mathbb{T} \times \mathbb{R} \rightarrow \mathbb{R}$ and $G: \mathbb{T} \times \mathbb{R}^{2} \rightarrow \mathbb{R}$ and

$$
u \mathrm{~F}(t, u)>0, \text { and } u G(t, u, v)>0 \text { for all } u \in \mathbb{R} \backslash\{0\}, v \in \mathbb{R}, t \geq t_{0} ;
$$

$\left(A_{3}\right)$ There exist $p, q: \mathbb{T} \rightarrow \mathbb{R}$ such that $p(t)-q(t)>0$ for all $t \geq t_{0}$ and

$$
\begin{aligned}
|F(t, u)| & \geq p(t)|u|, \\
|G(t, u, v)| & \leq q(s)|u| \text { for } u, v \neq 0 \text { for all } u, v \in \mathbb{R} \backslash\{0\}, t \geq t_{0} ;
\end{aligned}
$$

$\left(A_{4}\right) \int_{t_{0}}^{\infty}\left(\frac{1}{r(t)}\right) \Delta t=\infty$;

$\left(A_{5}\right) \int_{t_{0}}^{\infty}\left(\frac{1}{r(t)}\right) \Delta t<\infty$.

Recall that a solution of (1.2) is a nontrivial real function $x(t)$ such that $x(t) \in C_{r d}^{1}\left[t_{x}, \infty\right)_{\mathbb{T}}$, and $r(t) x^{\Delta}(t) \in C_{r d}^{1}\left[t_{x}, \infty\right)_{\mathbb{T}}$ for $t_{x} \geq t_{0}$ and satisfying equation (1.2) for $t \geq t_{x}$. Our attention is restricted to those solutions of (1.2) which exist on some half line $\left[t_{x}, \infty\right)_{\mathbb{T}}$ and satisfy $\sup \left\{|x(t)|: t>t_{1}\right\}>0$ for any $t_{1} \geq t_{x}$. A solution $x(t)$ of (1.2) is said to be oscillatory if it is neither eventually positive nor eventually negative. Otherwise it is called nonoscillatory equation (1.2) is said to be oscillatory if all its solutions are oscillatory.

We note that Equation (1.2) in its general form covers several different types of differential and difference equations depending on the choice of the time scale $\mathbb{T}$. For example, when $\mathbb{T}=\mathbb{R}$, we have $\sigma(t)=t, \mu(t)=0, x^{\Delta}(t)=x^{\prime}(t)$ and (1.2) becomes the second-order nonlinear perturbed differential equation

$$
\left(r(t) x^{\prime}(t)\right)^{\prime}+F(t, x(t))=G\left(t, x(t), x^{\prime}(t)\right), \quad t \in\left[t_{0}, \infty\right)_{\mathbb{T}} .
$$


When $\mathbb{T}=\mathbb{Z}$, we have $\sigma(t)=t+1, \mu(t)=1$,

$$
x^{\Delta}(t)=\Delta x(t)=x(t+1)-x(t),
$$

and (1.2) becomes the second-order nonlinear perturbed difference equation

$$
\Delta(r(t) \Delta x(t))+F(t, x(t+1))=G(t, x(t+1), \Delta x(t)), \quad t \in\left[t_{0}, \infty\right)_{\mathbb{T}} .
$$

When $\mathbb{T}=h \mathbb{Z}, h>0$, we have $\sigma(t)=t+h, \mu(t)=h$,

$$
x^{\Delta}(t)=\Delta_{h} x(t)=\frac{x(t+h)-x(t)}{h},
$$

and (1.2) becomes the second-order nonlinear perturbed difference equation

$$
\begin{aligned}
& \Delta_{h}\left(r(t) \Delta_{h} x(t)\right)+F(t, x(t+h)) \\
= & G\left(t, x(t+h), \Delta_{h} x(t+h)\right), t \in\left[t_{0}, \infty\right)_{\mathbb{T}} .
\end{aligned}
$$

When $\mathbb{T}=q^{\mathbb{N}}=\left\{t: t=q^{k}, k \in \mathbb{N}, q>1\right\}$, we have $\sigma(t)=q t, \mu(t)=(q-1) t$,

$$
x^{\Delta}(t)=\Delta_{q} x(t)=\frac{x(q t)-x(t)}{(q-1) t},
$$

and (1.2) becomes the second-order $q$-perturbed difference equation

$$
\Delta_{q}\left(r(t) \Delta_{q} x(t)\right)+F(t, x(q t))=G\left(t, x(q t), \Delta_{q} x(q t)\right), \quad t \in\left[t_{0}, \infty\right)_{\mathbb{T}} .
$$

When $\mathbb{T}=N_{0}^{2}=\left\{t^{2}: t \in \mathbb{N}_{0}\right\}$, we have $\sigma(t)=(\sqrt{t}+1)^{2}$ and $\mu(t)=1+2 \sqrt{t}$,

$$
\Delta_{N} x(t)=\frac{x\left((\sqrt{t}+1)^{2}\right)-x(t)}{1+2 \sqrt{t}},
$$

and (1.2) becomes the second-order nonlinear perturbed difference equation

$$
\begin{aligned}
& \Delta_{N}\left(r(t) \Delta_{N} x(t)\right)+F\left(t, x\left((\sqrt{t}+1)^{2}\right)\right) \\
= & G\left(t, x\left((\sqrt{t}+1)^{2}\right),\left(\Delta_{N} x\right)^{\sigma}\right), \quad t \in\left[t_{0}^{2}, \infty\right)_{\mathbb{T}} .
\end{aligned}
$$

When $\mathbb{T}=T_{n}=\left\{t_{n}: n \in \mathbb{N}_{0}\right\}$, where the $t_{n}$ are the so-called harmonic numbers defined by

$$
t_{0}=0, \quad t_{n}=\sum_{k=1}^{n} \frac{1}{k}, \quad n \in \mathbb{N}_{0}
$$

we have $\mu\left(t_{n}\right)=\frac{1}{n+1}$,

$$
x^{\Delta}(t)=\Delta_{t_{n}} x\left(t_{n}\right)=(n+1) \Delta_{t_{n}} x\left(t_{n}\right),
$$

and (1.2) becomes the second-order nonlinear perturbed difference equation

$$
\begin{aligned}
& \Delta_{t_{n}}\left(r\left(t_{n}\right) \Delta_{t_{n}} x\left(t_{n}\right)\right)+F\left(t_{n}, x\left(\sigma\left(\left(t_{n}\right)\right)\right)\right. \\
= & G\left(\sigma\left(t_{n}\right), x\left(\sigma\left(t_{n}\right)\right), \Delta_{t_{n}} x\left(\sigma\left(t_{n}\right)\right)\right), t_{n} \in[0, \infty)_{\mathbb{T}} .
\end{aligned}
$$


The paper is organized as follows: In the next section we present some basic definitions concerning the calculus on time scales. In Section 3, by using the new substitution

$$
w(t):=a(t)\left[\frac{r(t) x^{\Delta}(t)}{x(t)}+r(t) g(t)\right] \text { for } t \geq t_{1}>0,
$$

where $a(t)$ and $g(t)$ are given $r d$-continuous functions, we establish some new sufficient conditions which ensure that every solution of (1.2) oscillates or converges to zero. This technique can introduce some new sufficient conditions for oscillation and can be applied on different equations which cannot be covered by the results established by the Riccati technique. Our results in this paper improve the oscillation results given by Bohner and Saker [9] for dynamic equation (1.2) when $\gamma=1$. In Section 4 some examples are considered to illustrate our main results. To the best of our knowledge no results are given by this technique for perturbed dynamic equations before.

\section{Some preliminaries on time scales}

A time scale $\mathbb{T}$ is an arbitrary nonempty closed subset of the real numbers $\mathbb{R}$. Since we are interested in the oscillatory and asymptotic behavior of solutions near infinity, we assume that $\sup \mathbb{T}=\infty$. We define the forward jump operator on a time scale $\mathbb{T}$ by

$$
\sigma(t):=\inf \{s \in \mathbb{T}: s>t\} .
$$

A point $t \in \mathbb{T}$ is said to be right-dense if $\sigma(t)=t$, and right-scattered if $\sigma(t)>t$. The graininess function $\mu$ is defined by $\mu(t):=\sigma(t)-t$ for $t \in \mathbb{T}$. We define the time scale interval $[a, \infty)_{\mathbb{T} T}$ by

$$
[a, \infty)_{\mathbb{T}}:=[a, \infty) \cap \mathbb{T} .
$$

For a function $f: \mathbb{T} \rightarrow \mathbb{R}$, the (delta) derivative $f^{\Delta}(t)$ of $f$ at $t \in \mathbb{T}$ can be defined by

$$
f^{\Delta}(t)=\lim _{s \rightarrow t} \frac{f(t)-f(s)}{t-s},
$$

if $\sigma(t)=t$ (in this limit and others in this paper $s$ just takes on values in the time scale $\mathbb{T}$ ) and

$$
f^{\Delta}(t)=\frac{f(\sigma(t))-f(t)}{\mu(t)}
$$

if $f$ is continuous at $t$ and $\sigma(t)>t$. A function $f: \mathbb{T} \rightarrow \mathbb{R}$ is said to be $r d$ continuous if it is continuous at each right-dense point and at all left-dense points left hand limits exist and are finite. If $f$ is differentiable at $t$, then a useful formula is

$$
f^{\sigma}(t)=f(t)+\mu(t) f^{\Delta}(t), \quad \text { where } \quad f^{\sigma}(t):=(f \circ \sigma)(t)=f(\sigma(t)) .
$$

Assuming $f$ and $g$ are delta differentiable we will make use of the product rule

$$
(f(t) g(t))^{\Delta}=f^{\Delta}(t) g(t)+f(\sigma(t)) g^{\Delta}(t)=f(t) g^{\Delta}(t)+f^{\Delta}(t) g(\sigma(t)),
$$


and the quotient rule

$$
\left(\frac{f}{g}\right)^{\Delta}(t)=\frac{f^{\Delta}(t) g(t)-f(t) g^{\Delta}(t)}{g(t) g(\sigma(t))}
$$

provided $g(t) g^{\sigma}(t) \neq 0$. For $a, b \in \mathbb{T}$ and a differentiable function $f$, the Cauchy (delta) integral of $f^{\Delta}$ is defined by

$$
\int_{a}^{b} f^{\Delta}(t) \Delta t=f(b)-f(a) .
$$

The integration by parts formula reads

$$
\int_{a}^{b} f^{\Delta}(t) g(t) \Delta t=\left.f(t) g(t)\right|_{a} ^{b}-\int_{a}^{b} f^{\sigma}(t) g^{\Delta}(t) \Delta t
$$

and we define the improper integral

$$
\int_{a}^{\infty} f(s) \Delta s=\lim _{t \rightarrow \infty} \int_{a}^{t} f(s) \Delta s .
$$

We now give some examples of what we have discussed so far. First, if $\mathbb{T}=\mathbb{R}$, we have

$$
\sigma(t)=t, \quad \mu(t) \equiv 0, \quad f^{\Delta}(t)=f^{\prime}(t), \quad \text { and } \quad \int_{a}^{b} f(t) \Delta t=\int_{a}^{b} f(t) d t .
$$

If $\mathbb{T}=\mathbb{Z}$, we have

$$
\sigma(t)=t+1, \quad \mu(t) \equiv 1, \quad f^{\Delta}(t)=\Delta f(t), \quad \text { and } \quad \int_{a}^{b} f(t) \Delta t=\sum_{t=a}^{b-1} f(t) .
$$

For $\mathbb{T}=h \mathbb{Z}, h>0$, we have $\sigma(t)=t+h, \mu(t)=h$,

$$
f^{\Delta}(t)=\Delta_{h} f(t):=\frac{f(t+h)-f(t)}{h}, \text { and } \int_{a}^{b} f(t) \Delta t=\sum_{k=0}^{\frac{b-a-h}{h}} f(a+k h) h .
$$

Finally, if $\mathbb{T}=\varrho^{\mathbb{N}_{0}}=\left\{t: t=\varrho^{k}, k \in \mathbb{N}_{0}\right\}$, where $\varrho>1$, we have $\sigma(t)=\varrho t$, $\mu(t)=(\varrho-1) t$

$$
x^{\Delta}(t)=\frac{x(\varrho t)-x(t)}{(\varrho-1) t}, \quad \text { and } \quad \int_{a}^{\infty} f(t) \Delta t=\sum_{k=0}^{\infty} f\left(\varrho^{k}\right) \mu\left(\varrho^{k}\right) .
$$

\section{Main results}

In this section, we establish some sufficient conditions for oscillation of all solutions of $(1.2)$ when $\left(A_{4}\right)$ holds and when $\left(A_{5}\right)$ holds we establish some sufficient conditions which ensure that every solution of (1.2) oscillates or converges to zero. In the following, we assume that $\sigma(\rho(t))=\rho(\sigma(t)))$ and 
$a(t)>0$ for $t \geq t_{0}$ is a given $r d$-continuous $\Delta$-differentiable function such that $a^{\sigma}(t)\left(\frac{r(t) a^{\Delta}(t)}{a(t)}\right) \leq M$, and define

$$
\psi(t):=a(t)\left[p(s)-q(s)-(r(s) g(s))^{\Delta}+r(s)\left(\frac{(r g)^{\sigma}}{r(s)}\right)^{2}\right],
$$

where $(r g)^{\sigma}=-\frac{r(t) a^{\Delta}(t)}{2 a(t)}$.

First, we consider the case when $\left(A_{4}\right)$ holds.

Theorem 3.1. Assume that $\left(A_{1}\right)-\left(A_{4}\right)$ hold. If

$$
\limsup _{t \rightarrow \infty} \int_{t_{0}}^{t} \psi(s) \Delta s=\infty \text {, }
$$

then every solution of equation $(1.2)$ is oscillatory on $\left[t_{0}, \infty\right)_{\mathbb{T}}$.

Proof. Suppose to the contrary that $x(t)$ is a nonoscillatory solution of (1.2), and let $t_{1} \geq t_{0}$ be such that $x(t) \neq 0$ for all $t \geq t_{1}$. Without loss of generality, we may assume that $x(t)>0$ for $t \geq t_{0}$. In view of (1.2) and $\left(A_{3}\right)$ we have

$$
\left(r(t) x^{\Delta}(t)\right)^{\Delta} \leq-(p(t)-q(t)) x^{\sigma}<0 \text { for } t \geq t_{1},
$$

and so $r(t) x^{\Delta}(t)$ is an eventually decreasing function. We first show that $r(t) x^{\Delta}(t)$ is eventually nonnegative. Indeed, since $p(t)-q(t)$ is a positive function, the decreasing function $r(t) x^{\Delta}(t)$ is either eventually positive or eventually negative. Suppose there exists an integer $t_{2} \geq t_{1}$ such that $r\left(t_{1}\right) x^{\Delta}\left(t_{1}\right)=c<0$. So that from (3.2) we have $r(t) x^{\Delta}(t)<r\left(t_{1}\right) x^{\Delta}\left(t_{1}\right)=c$ for $t \geq t_{2}$, and then we get

This implies by $\left(A_{4}\right)$, that

$$
x^{\Delta}(t) \leq c\left(\frac{1}{r(t)}\right)
$$

$$
x(t) \leq x\left(t_{2}\right)+c \int_{t_{2}}^{t}\left(\frac{1}{r(s)}\right) \Delta s \rightarrow-\infty \text { as } t \rightarrow \infty,
$$

which contradicts the fact that $x(t)>0$ for all $t \geq t_{1}$. Hence $r(t) x^{\Delta}(t)$ is eventually nonnegative. Therefore, we have

$$
x(t)>0, x^{\Delta}(t) \geq 0,\left(r(t) x^{\Delta}(t)\right)^{\Delta}<0 \text { for } t \geq t_{1} .
$$

Define the function $w(t)$ by

$$
w(t):=a(t)\left[\frac{r(t) x^{\Delta}(t)}{x(t)}+r(t) g(t)\right] \quad \text { for } t \geq t_{1} .
$$

Then, we have

$$
\begin{aligned}
w^{\Delta}(t) & =a^{\Delta}(t)\left[\frac{r(t) x^{\Delta}(t)}{x(t)}+r(t) g(t)\right]^{\sigma}+a(t)\left[\frac{r(t) x^{\Delta}(t)}{x(t)}+r(t) g(t)\right]^{\Delta} \\
& =\frac{a^{\Delta}(t)}{a^{\sigma}} w^{\sigma}+a(t)[r(t) g(t)]^{\Delta}+a(t)\left[\frac{r(t) x^{\Delta}(t)}{x(t)}\right]^{\Delta} .
\end{aligned}
$$


Now, since

$$
\begin{aligned}
a(t)\left[\frac{r(t) x^{\Delta}(t)}{x(t)}\right]^{\Delta} & =a(t)\left[\left(r x^{\Delta}\right)^{\sigma}\left(\frac{1}{x(t)}\right)^{\Delta}+\frac{1}{x(t)}\left(r(t) x^{\Delta}(t)\right)^{\Delta}\right] \\
& =\left[-a(t) \frac{1}{x(t)}\left(r(t) x^{\Delta}(t)\right)^{\Delta}-a(t)\left(r x^{\Delta}\right)^{\sigma} \frac{x^{\Delta}(t)}{x(t) x^{\sigma}}\right],
\end{aligned}
$$

we have from (3.2) that

$$
a(t)\left[\frac{r(t) x^{\Delta}(t)}{x(t)}\right]^{\Delta} \leq\left[-a(t)[p(t)-q(t)] \frac{x^{\sigma}}{x(t)}-a(t)\left(r x^{\Delta}\right)^{\sigma} \frac{x^{\Delta}(t)}{x(t) x^{\sigma}}\right] .
$$

From (3.3), since $r x^{\Delta}(t)$ is nonincreasing and $x(t)$ is nondecreasing, we have $r x^{\Delta}(t) \geq\left(r x^{\Delta}\right)^{\sigma}$ and $x^{\sigma} \geq x(t)$, and this implies that

$$
a(t)\left[\frac{r(t) x^{\Delta}(t)}{x(t)}\right]^{\Delta} \leq\left[-a(t)[p(t)-q(t)]-\frac{a(t)}{r(t)}\left(\left(\frac{r x^{\Delta}}{x}\right)^{\sigma}\right)^{2}\right] .
$$

From the definition of $w(t)$, we see that

$$
\left(\left(\frac{r x^{\Delta}}{x}\right)^{\sigma}\right)^{2}=\left[\frac{w^{\sigma}}{a^{\sigma}}-r^{\sigma} g^{\sigma}\right]^{2}=\left[\left(\frac{w^{\sigma}}{a^{\sigma}}\right)^{2}-2 \frac{w^{\sigma}}{a^{\sigma}} r^{\sigma} g^{\sigma}+\left(r^{\sigma} g^{\sigma}\right)^{2}\right]
$$

Substituting from the last equation in (3.5), we get

$$
\begin{aligned}
& a(t)\left[\frac{r(t) x^{\Delta}(t)}{x(t)}\right]^{\Delta} \\
\leq & -a(t)[p(t)-q(t)]-\frac{a(t)}{r(t)}\left(\frac{w^{\sigma}}{a^{\sigma}}\right)^{2}+2 \frac{a(t) r^{\sigma} g^{\sigma}}{a^{\sigma} r(t)} w^{\sigma}-\frac{a(t)}{r(t)}\left(r^{\sigma} g^{\sigma}\right)^{2} .
\end{aligned}
$$

From (3.4) and (3.6), we have

$$
\begin{aligned}
w^{\Delta}(t) \leq & -a(t)[p(t)-q(t)]+a(t)(r(t) g(t))^{\Delta}-\frac{a(t)}{r(t)}\left(\frac{w^{\sigma}}{a^{\sigma}}\right)^{2} \\
& +\frac{a^{\Delta}(t)}{a^{\sigma}} w^{\sigma}+2 \frac{a(t) r^{\sigma} g^{\sigma}}{a^{\sigma} r(t)} w^{\sigma}-\frac{a(t)}{r(t)}\left(r^{\sigma} g^{\sigma}\right)^{2}
\end{aligned}
$$

Using the fact that $g^{\sigma}=-\frac{r(t) a^{\Delta}(t)}{2 a(t) r^{\sigma}}$, we see that

$$
\begin{aligned}
w^{\Delta}(t) \leq & -\frac{a(t)}{r(t)\left(a^{\sigma}\right)^{2}}\left(w^{\sigma}\right)^{2} \\
& -a(t)\left[p(t)-q(t)-(r(t) g(t))^{\Delta}+r(t)\left(\frac{r^{\sigma} g^{\sigma}}{r(t)}\right)^{2}\right] .
\end{aligned}
$$

This gives

$$
w^{\Delta}(t)<-a(t)\left[p(t)-q(t)-(r(t) g(t))^{\Delta}+r(t)\left(\frac{r^{\sigma} g^{\sigma}}{r(t)}\right)^{2}\right] .
$$


Integrating (3.9) from $t_{1}$ to $t\left(t \geq t_{1}\right)$, we find that

$$
\int_{t_{1}}^{t} a(s)\left[p(s)-q(s)-(r(s) g(s))^{\Delta}+r(s)\left(\frac{r^{\sigma} g^{\sigma}}{r(s)}\right)^{2}\right] \Delta s<w\left(t_{1}\right)-w(t) .
$$

Now from the definition of $w(t)$, we have

$$
w(t) \geq-a(t)\left(\frac{r^{\rho} a^{\Delta}}{2 a}\right)^{\rho} \quad \text { for } t \geq t_{1} .
$$

Using the fact that $a(t)\left(\frac{r^{\rho} a^{\Delta}}{a}\right)^{\rho} \leq M$, we have

$$
w(t)>-\frac{1}{2} M
$$

and therefore, it follows that the right side of (3.10) is bounded above. This is a contrary to (3.1) and completes the proof.

Remark 3.1. From Theorem 3.1, we can obtain different conditions for oscillation of all solutions of (1.2) by different choices of $a(t)$. For instance, if we put $a(t)=t, t \geq t_{0}$, then $g^{\sigma}=-r(t) / 2 t r^{\sigma}$ and by Theorem 3.1, we have the following oscillation result.

Corollary 3.1. Assume that $\left(A_{1}\right)-\left(A_{4}\right)$ hold. If

$$
\limsup _{t \rightarrow \infty} \int_{t_{0}}^{t} s\left[p(s)-q(s)+\frac{1}{4 s^{2}} r(s)+\left(\frac{r^{\rho}}{2 \rho(s)}\right)^{\Delta}\right] \Delta s=\infty
$$

then every solution of equation $(1.2)$ is oscillatory on $\left[t_{0}, \infty\right)_{\mathbb{T}}$.

Theorem 3.2. Assume that $\left(A_{1}\right)-\left(A_{4}\right)$ hold. If

$\limsup _{t \rightarrow \infty} \frac{1}{t^{m}} \int_{t_{0}}^{t}\left[(t-s)^{m} \psi(s)-\frac{m^{2}(t-\sigma(s))^{2 m-2}}{4(t-s)^{m}} \frac{r(s)\left(a^{\sigma}\right)^{2}}{a(s)}\right] \Delta s=\infty, m>1$,

then every solution of $(1.2)$ is oscillatory on $\left[t_{0}, \infty\right)_{\mathbb{T}}$.

Proof. Suppose to the contrary that $x(t)$ is a nonoscillatory solution of (1.2), and let $t_{1} \geq t_{0}$ be such that $x(t) \neq 0$ for all $t \geq t_{1}$. Without loss of generality we assume that $x(t)>0$ for $t \geq t_{0}$ and let $w(t)$ be as given in Theorem 3.1 and proceeding to obtain (3.8). Multiplying (3.8) by $(t-s)^{m}$ and integrating from $t_{1}$ to $t$, we have

$$
\begin{aligned}
\int_{t_{1}}^{t}(t-s)^{m} \psi(s) \Delta s \leq & -\int_{t_{1}}^{t}(t-s)^{m} \frac{a(s)}{r(s)\left(a^{\sigma}\right)^{2}}\left(w^{\sigma}\right)^{2} \Delta s \\
& -\int_{t_{1}}^{t}(t-s)^{m} w^{\Delta}(s) \Delta s .
\end{aligned}
$$


An integration by parts formula, the last term in (3.12) leads to

(3.13) $-\int_{t_{1}}^{t}(t-s)^{m} w^{\Delta}(s) \Delta s=-\left.(t-s)^{m} w(s)\right|_{t_{1}} ^{t}+\int_{t_{1}}^{t}\left((t-s)^{m}\right)^{\Delta} w^{\sigma} \Delta s$.

Now, we prove that

$$
\left((t-s)^{m}\right)^{\Delta} \leq-m(t-\sigma(s))^{m-1} .
$$

We consider the following two cases:

Case 1: If $\mu(t)=0$, then

$$
\left((t-s)^{m}\right)^{\Delta}=-m(t-s)^{m-1} .
$$

Case 2: Let $\mu(t) \neq 0$, then we have

$$
\begin{aligned}
\left((t-s)^{m}\right)^{\Delta} & =\frac{1}{\mu(s)}\left[\left((t-\sigma(s))^{m}\right)-\left((t-s)^{m}\right)\right] \\
& =-\frac{1}{\sigma(s)-s}\left[\left((t-s)^{m}\right)-\left((t-\sigma(s))^{m}\right)\right] .
\end{aligned}
$$

Using Hardy, Littlewood and Polya inequality (cf. [17])

$$
x^{m}-y^{m} \geq m y^{m-1}(x-y) \text { for all } x \geq y>0 \text { and } m \geq 1,
$$

we have

$$
\left[\left((t-s)^{m}\right)-\left((t-\sigma(s))^{m}\right)\right] \geq m\left((t-\sigma(s))^{m-1}(\sigma(s)-s),\right.
$$

and then

$$
\left((t-s)^{m}\right)^{\Delta} \leq-m\left((t-\sigma(s))^{m-1} .\right.
$$

Then from (3.15) and (3.16) since in general case $\sigma(s) \geq s$, we see that (3.14) holds. From (3.12)-(3.14), we have

$$
\begin{aligned}
& \int_{t_{1}}^{t}(t-s)^{m} \psi(s) \Delta s \\
\leq & -\left.(t-s)^{m} w(s)\right|_{t_{1}} ^{t}-\int_{t_{1}}^{t}\left[\frac{m(t-\sigma(s))^{m-1}}{2(t-s)^{\frac{m}{2}}} \sqrt{\frac{r(s)\left(a^{\sigma}\right)^{2}}{a(s)}}\right]^{2} \Delta s \\
& -\int_{t_{1}}^{t}\left[(t-s)^{\frac{m}{2}} \sqrt{\frac{a(s)}{r(s)\left(a^{\sigma}\right)^{2}}} w^{\sigma}-\frac{m(t-\sigma(s))^{m-1}}{2(t-s)^{\frac{m}{2}}} \sqrt{\frac{r(s)\left(a^{\sigma}\right)^{2}}{a(s)}}\right]^{2} \Delta s .
\end{aligned}
$$

Then

$$
\int_{t_{1}}^{t}\left[(t-s)^{m} \psi(s)-\frac{m^{2}(t-\sigma(s))^{2 m-2}}{4(t-s)^{m}} \frac{r(s)\left(a^{\sigma}\right)^{2}}{a}\right] \Delta s<\left(t-t_{1}\right)^{m} w\left(t_{1}\right),
$$

which implies that

$$
\frac{1}{t^{m}} \int_{t_{1}}^{t}\left[(t-s)^{m} \psi(s)-\frac{m^{2}(t-s)^{m-2}}{4} \frac{r(s)\left(a^{\sigma}\right)^{2}}{a(s)}\right] \Delta s<\left(\frac{t-t_{1}}{t}\right)^{m} w\left(t_{1}\right) \text {. }
$$


Hence,

$\limsup _{t \rightarrow \infty} \frac{1}{t^{m}} \int_{t_{1}}^{t}\left[(t-s)^{m} \psi(s)-\frac{m^{2}(t-s)^{m-2}}{4} \frac{r(s)\left(a^{\sigma}\right)^{2}}{a(s)}\right] \Delta s \rightarrow$ finite number, which contradicts (3.11). The proof is complete.

We define $\Re$ by $H \in \Re$ provided $H:\left[t_{0}, \infty\right)_{\mathbb{T}} \times\left[t_{0}, \infty\right)_{\mathbb{T}} \rightarrow \mathbb{R}$ satisfies

$$
H(t, t) \geq 0, \quad t \geq t_{0}, \quad H(t, s)>0, \quad t>s \geq t_{0},
$$

$H^{\Delta_{s}}(t, s) \leq 0$ for $t \geq s \geq a$, and for each fixed $t, H(t, s)$ is right-dense continuous with respect to $s$. An important example of $H$ is $H(t, s)=(t-s)^{(k)}$, where $t^{(k)}=t(t-1) \cdots(t-k+1), t^{(0)}=1$.

Theorem 3.3. Assume that $\left(A_{1}\right)-\left(A_{4}\right)$ hold, and let $h, H$ be rd-continuous functions such that $H$ belongs to the class $\Re$ and

$$
h(t, s)=-\frac{H^{\Delta_{s}}(t, s)}{\sqrt{H(t, s)}} .
$$

If

$$
\limsup _{t \rightarrow \infty} \frac{1}{H\left(t, t_{0}\right)} \int_{t_{0}}^{t}\left[H(t, s) \psi(s)-\frac{r(s)\left(a^{\sigma}\right)^{2}}{4 a(s)} h^{2}(t, s)\right] \Delta s=\infty
$$

then every solution of equation $(1.2)$ is oscillatory on $\left[t_{0}, \infty\right)_{\mathbb{T}}$.

Proof. Suppose to the contrary that $x(t)$ is a nonoscillatory solution of (1.2), and let $t_{1} \geq t_{0}$ be such that $x(t) \neq 0$ for all $t \geq t_{1}$. Without loss of generality we assume that $x(t)>0$ for $t \geq t_{0}$ and let $w(t)$ be as given in Theorem 3.1 and proceeding to obtain (3.8). Multiplying (3.8) by $H(t, s)$ and integrating from $t_{1}$ to $t$, we have

$$
\int_{t_{1}}^{t} H(t, s) \psi(s) \Delta s \leq-\int_{t_{1}}^{t} H(t, s) w^{\Delta}(s) \Delta s-\int_{t_{1}}^{t} \frac{H(t, s) a(s)}{r(s)\left(a^{\sigma}\right)^{2}}\left(w^{\sigma}\right)^{2} \Delta s .
$$

Using integration by parts formula (2.6), we have

$$
\begin{aligned}
\int_{t_{1}}^{t} H(t, s) w^{\Delta}(s) \Delta s & =\left.H(t, s) w(s)\right|_{t_{1}} ^{t}-\int_{t_{1}}^{t} H^{\Delta_{s}}(t, s) w^{\sigma} \Delta s \\
& =-H\left(t, t_{1}\right) w\left(t_{1}\right)-\int_{t_{1}}^{t} H^{\Delta_{s}}(t, s) w^{\sigma} \Delta s
\end{aligned}
$$

where $H(t, t)=0$. Substituting from (3.20) in (3.19) and using (3.17), we get

$$
\begin{aligned}
\int_{t_{1}}^{t} H(t, s) \psi(s) \Delta s \leq & H\left(t, t_{1}\right) w\left(t_{1}\right)-\int_{t_{1}}^{t} h(t, s) \sqrt{H(t, s)} w^{\sigma} \Delta s \\
& -\int_{t_{1}}^{t} \frac{H(t, s) a(s)}{r(s)\left(a^{\sigma}\right)^{2}}\left(w^{\sigma}\right)^{2} \Delta s .
\end{aligned}
$$


Therefore

$$
\begin{aligned}
& \int_{t_{1}}^{t} H(t, s) \psi(s) \Delta s \\
\leq & H\left(t, t_{1}\right) w\left(t_{1}\right)+\int_{t_{1}}^{t} \frac{r(s)\left(a^{\sigma}\right)^{2} h^{2}(t, s)}{4 a(s)} \Delta s \\
& -\int_{t_{1}}^{t}\left[\sqrt{\frac{H(t, s) a(s)}{\left(r(s)\left(a^{\sigma}\right)^{2}\right)}} w^{\sigma}+\frac{h(t, s) \sqrt{r(s)\left(a^{\sigma}\right)^{2} /(a(s))}}{2}\right]^{2} \Delta s .
\end{aligned}
$$

Then, for all $t \geq t_{1}$ we have

$$
\int_{t_{1}}^{t}\left[H(t, s) \psi(s)-\frac{r(s)\left(a^{\sigma}\right)^{2} h^{2}(t, s)}{4 a(s)}\right] \Delta s<H\left(t, t_{1}\right) w\left(t_{1}\right),
$$

and this implies that

$$
\frac{1}{H\left(t, t_{1}\right)} \int_{t_{1}}^{t}\left[H(t, s) \psi(s)-\frac{r(s)\left(a^{\sigma}\right)^{2}}{4 a(s)} h^{2}(t, s)\right] \Delta s<w\left(t_{1}\right)
$$

for all large $t$, which contradicts (3.18). The proof is complete.

As an immediate consequence of Theorem 3.3 by putting $a(t)=1$, we get the following results

Theorem 3.4. Assume that $\left(A_{1}\right)-\left(A_{4}\right)$ hold, and let $h, H$ be rd-continuous functions such that $H$ belongs to the class $\Re$ such that (3.17) holds. If

$$
\limsup _{t \rightarrow \infty} \frac{1}{H\left(t, t_{0}\right)} \int_{t_{0}}^{t}\left[H(t, s)[p(s)-q(s)]-\frac{r(s) h^{2}(t, s)}{4}\right] \Delta s=\infty,
$$

then every solution of equation $(1.2)$ is oscillatory on $\left[t_{0}, \infty\right)_{\mathbb{T} T}$.

\section{Next, we consider the case when $\left(A_{5}\right)$ holds.}

In the following, we consider the case when $\left(A_{5}\right)$ holds and establish some sufficient conditions which insure that every solution of (1.2) oscillates or converges to zero. We start with the following auxiliary result, which the proof is similar as in [9] and hence is omitted.

Lemma 3.1. Assume that $\left(A_{1}\right)-\left(A_{3}\right)$ and $\left(A_{5}\right)$ hold, and

$$
\int_{t_{0}}^{\infty} \frac{1}{r(t)} \int_{t_{0}}^{t}[p(s)-q(s)] \Delta s \Delta t=\infty .
$$

Suppose that $x$ is a nonoscillatory solution of (1.2) such that there exists $t_{1} \in \mathbb{T}$ with $x(t) x^{\Delta}(t)<0$ for all $t \geq t_{1}$. Then

$$
\lim _{t \rightarrow \infty} x(t) \text { exists and is zero. }
$$

Using Lemma 3.1, we can derive the following criteria. 
Theorem 3.5. Assume that $\left(A_{1}\right)-\left(A_{3}\right),\left(A_{5}\right)$ and (3.25) hold. If there exists a positive differentiable function a $(t)$ such that (3.1) holds. Then every solution $x(t)$ of (1.2) oscillates or $\lim _{t \rightarrow \infty} x(t)=0$.

Proof. Assume that $x$ is a nonoscillatory solution of (1.2). Hence $x$ is either eventually positive or eventually negative, i.e., there exists $t_{1} \geq t_{0}$ with $x(t)>0$ for all $t \geq t_{1}$ or $x(t)<0$ for all $t \geq t_{1}$. Without loss of generality we assume that $x(t)>0$ for $t \geq t_{1}$. From (1.2) and $\left(A_{3}\right)$, we have

$$
\left(r(t) x^{\Delta}(t)\right)^{\Delta}=-[p(t)-q(t)] x^{\sigma}<0,
$$

and so $r(t) x^{\Delta}(t)$ is an eventually decreasing function and either $x^{\Delta}(t)$ is eventually positive or eventually negative. If $x^{\Delta}(t)$ is eventually positive we can derive a contradiction as in Theorem 3.1. If $x^{\Delta}(t)$ is eventually negative we have from Lemma 3.1 that $\lim _{t \rightarrow \infty} x(t)=0$. This completes the proof.

Theorem 3.6. Assume that $\left(A_{1}\right)-\left(A_{3}\right),\left(A_{5}\right)$ and $(3.25)$. If there exists a positive differentiable function $a(t)$ such that (3.11) holds. Then every solution $x(t)$ of (1.2) oscillates or $\lim _{t \rightarrow \infty} x(t)=0$.

Theorem 3.7. Assume that $\left(A_{1}\right)-\left(A_{3}\right)$, $\left(A_{5}\right)$ and $(3.25)$ hold, $h, H$ are $r d$ continuous functions such that $H$ belongs to the class $\Re$ and (3.17) holds. If there exists a positive differentiable function $a(t)$ such that (3.18) holds. Then every solution $x(t)$ of (1.2) oscillates or $\lim _{t \rightarrow \infty} x(t)=0$.

\section{Examples}

In this section, we give some examples to illustrate our main results.

Example 4.1. Consider the following second order dynamic equation (4.1)

$$
x^{\prime \prime}(t)+\left(\frac{\gamma}{t^{2}}+t^{2}(x(t))^{2}\right) x(t)=\frac{d(x(t))^{3}}{t^{2}\left(1+(x(t))^{2}\right)\left(1+\left((x(t))^{\Delta}\right)^{2}\right)}, t \in\left[t_{0}, \infty\right)_{\mathbb{T}},
$$

where $\mathbb{T}=\mathbb{R}$ with $t_{0}>0, \gamma$ and $d$ are positive constants. Here $r(t) \equiv 1$, $p(t):=\frac{\gamma}{t^{2}}$, and $q(t):=\frac{d}{t^{2}}$. It is easy to see that the assumptions $\left(A_{1}\right)-\left(A_{4}\right)$ hold and $p(t)-q(t)=\frac{\gamma-d}{t^{2}}$. We will apply Corollary 3.1. Note

$$
\begin{aligned}
& \limsup _{t \rightarrow \infty} \int_{t_{0}}^{t} s\left[p(s)-q(s)+\frac{1}{4 s^{2}} r(s)+\left(\frac{r^{\rho}}{2 \rho(s)}\right)^{\Delta}\right] \Delta s \\
= & \limsup _{t \rightarrow \infty} \int_{t_{0}}^{t}\left[\frac{\gamma-d}{s}+\frac{1}{4 s}+\frac{s}{2}\left(\frac{1}{s}\right)^{\prime}\right] d s \\
\geq & \limsup _{t \rightarrow \infty} \int_{t_{0}}^{t}\left[\frac{\gamma-d}{s}+\frac{1}{4 s}+\frac{s}{2}\left(\frac{1}{s}\right)^{\prime}\right] d s=\limsup _{t \rightarrow \infty} \int_{1}^{t}\left[\frac{\gamma-d}{s}+\frac{1}{4 s}-\frac{1}{2 s}\right] d s \\
\geq & \limsup _{t \rightarrow \infty} \int_{t_{0}}^{t}\left[\frac{\gamma-d}{s}+\frac{1}{4 s}-\frac{1}{2 s}\right] d s=\limsup _{t \rightarrow \infty} \int_{1}^{t}\left[\frac{\gamma-d}{s}-\frac{1}{4 s}\right] d s=\infty,
\end{aligned}
$$


provided that $\gamma-d>1 / 4$. Hence every solution of (4.1) oscillates if $\gamma-d>\frac{1}{4}$.

Example 4.2. Consider the dynamic equation

$$
\left(\frac{1}{t} x^{\Delta}\right)^{\Delta}+\left(\frac{\alpha}{t^{3}}+t^{4}\left(x^{\sigma}\right)^{4}\right) x^{\sigma}=\frac{\beta\left(x^{\sigma}\right)^{5}}{t^{3}\left(1+\left(x^{\sigma}\right)^{4}\right)\left(1+\left(\left(x^{\sigma}\right)^{\Delta}\right)^{4}\right)},
$$

for $t \in\left[t_{0}, \infty\right)_{\mathbb{T}}$ is a time scale for $t_{0}>0, \alpha$ and $\beta$ are positive constants. Here $r(t) \equiv 1 / t, p(t):=\frac{\alpha}{t^{3}}$, and $q(t):=\frac{\beta}{t^{3}}$. It is easy to see that the assumptions $\left(A_{1}\right)-\left(A_{4}\right)$ hold and $p(t)-q(t)=\frac{\alpha-\beta}{t^{3}}$ for $t \in\left[t_{0}, \infty\right)_{\mathbb{T}}$. To apply Theorem 3.1, we choose $a(t)=t^{2}$, which implies that $g^{\sigma}=-\frac{r(s)}{r^{\sigma}} \frac{t+\sigma(t)}{2 t^{2}}$ and prove that

$$
\begin{aligned}
& \limsup _{t \rightarrow \infty} \int_{t_{0}}^{t} a(s)\left[p(s)-q(s)+\frac{r(s)}{4}\left(\frac{(s+\sigma(s)}{s^{2}}\right)^{2}-[r(t) g(t)]^{\Delta}\right] \Delta s \\
= & \limsup _{t \rightarrow \infty} \int_{t_{0}}^{t} s^{2}\left[\frac{\alpha-\beta}{s^{3}}+\frac{1}{4 s}\left(\frac{s+\sigma(s)}{s^{2}}\right)^{2}+\left(\frac{s+\rho(s)}{2 \rho^{3}}\right)^{\Delta}\right] \Delta s=\infty
\end{aligned}
$$

on different time scales. In the case when $\mathbb{T}=\mathbb{R}, \sigma(s)=\rho(s)=s$, and so we have $a^{\sigma}\left(\frac{r(t) a^{\Delta}(t)}{a(t)}\right)=a(t)\left(\frac{r(t) a^{\Delta}(t)}{a(t)}\right)=2$. For $t_{0}=1$, we obtain

$$
\begin{aligned}
& \limsup _{t \rightarrow \infty} \int_{t_{0}}^{t} s^{2}\left[\frac{\alpha-\beta}{s^{3}}+\frac{1}{4 s}\left(\frac{(s+\sigma(s))}{s^{2}}\right)^{2}+\left(\frac{\rho(s)+s}{2 \rho^{3}(s)}\right)^{\prime}\right] d s \\
= & \limsup _{t \rightarrow \infty} \int_{1}^{t} s^{2}\left[\frac{\alpha-\beta}{s^{3}}+\frac{1}{s^{3}}+\left(\frac{1}{2 s^{2}}\right)^{\prime}\right] d s \\
= & \limsup _{t \rightarrow \infty} \int_{1}^{t} s^{2}\left[\frac{\alpha-\beta}{s^{3}}\right] d s=\infty,
\end{aligned}
$$

provided that $\alpha-\beta>1$. Hence every solution of differential equation

$$
\left(\frac{1}{t} x^{\prime}(t)\right)^{\prime}+\left(\frac{\alpha}{t^{3}}+t^{4}(x(t))^{4}\right) x(t)=\frac{\beta(x(t))^{5}}{t^{3}\left(1+(x(t))^{4}\right)\left(1+\left((x(t))^{\prime}\right)^{4}\right)}, \quad t \geq 1,
$$

oscillates if $\alpha-\beta>1$. In the case $\mathbb{T}=\mathbb{N}$, for $t_{0}=2, \sigma(s)=s+1, \rho(s)=s-1$, and

$$
\begin{aligned}
& \limsup _{t \rightarrow \infty} \int_{t_{0}}^{t} s^{2}\left[\frac{\alpha-\beta}{s^{3}}+\frac{1}{s}\left(\frac{s+\sigma(s)}{2 s^{2}}\right)^{2}+\left(\frac{s+\rho(s)}{2 \rho^{3}}\right)^{\Delta}\right] \Delta s \\
= & \limsup _{t \rightarrow \infty} \sum_{s=2}^{t-1} s^{2}\left[\frac{\alpha-\beta}{s^{3}}+\frac{1}{s}\left(\frac{s+s+1}{2 s^{2}}\right)^{2}+\Delta\left(\frac{s-1+s}{2(s-1)^{3}}\right)\right]
\end{aligned}
$$




$$
\begin{aligned}
& =\limsup _{t \rightarrow \infty} \sum_{s=2}^{t-1} s^{2}\left[\frac{\alpha-\beta}{s^{3}}+\frac{1}{s}\left(\frac{2 s+1}{2 s^{2}}\right)^{2}+\Delta\left(\frac{2 s-1}{2(s-1)^{3}}\right)\right] \\
& =\limsup _{t \rightarrow \infty} \sum_{s=2}^{t-1} s^{2}\left[\frac{\alpha-\beta}{s^{3}}+\frac{1}{s}\left(\frac{2 s+1}{2 s^{2}}\right)^{2}+\frac{2 s+1}{2 s^{3}}-\frac{2 s-1}{2(s-1)^{3}}\right] \\
& =\limsup _{t \rightarrow \infty} \sum_{s=2}^{t-1} s^{2}\left[\frac{\alpha-\beta}{s^{3}}+\frac{1}{4 s^{5}}(2 s+1)^{2}+\frac{1}{2 s^{3}}+\frac{1}{s^{2}}-\frac{1}{2(s-1)^{3}}-\frac{1}{(s-1)^{2}}\right] \\
& \geq \limsup _{t \rightarrow \infty} \sum_{s=2}^{t-1} s^{2}\left[\frac{\alpha-\beta}{s^{3}}+\frac{1}{s^{3}}+\frac{1}{2 s^{3}}+\frac{1}{s^{2}}-\frac{1}{2(s-1)^{3}}-\frac{1}{(s-1)^{2}}\right] \\
& =\limsup _{t \rightarrow \infty} \sum_{s=2}^{t-1}\left[\frac{\alpha-\beta}{s}+\frac{1}{s}+\frac{1}{2 s}+1-\frac{s^{2}}{2(s-1)^{3}}-\frac{s^{2}}{(s-1)^{2}}\right] \\
& \qquad \sum_{s=2}^{\infty}\left[\frac{1}{2} \frac{2 \gamma s^{3}-6 \gamma s^{2}+6 \gamma s-2 \gamma-2 s^{3}-3 s^{2}+7 s-3}{s(s-1)^{3}}\right] \\
& =\operatorname{signum}(\gamma-1) \infty-\frac{5}{2}-\frac{1}{2} \zeta(3)-\frac{1}{3} \pi^{2}-\frac{1}{2}(2 \gamma-2)(1-\text { gamma }) \\
& =\infty,
\end{aligned}
$$

provided that $\gamma=\alpha-\beta>1$, where $\zeta$ is Riemann Zeta function and

$$
\text { gamma }=\lim _{n \rightarrow \infty} \sum_{m=1}^{n}\left[\frac{1}{m}-\ln n\right]=\infty .
$$

Then every solution of difference equation

$$
\begin{aligned}
& \Delta\left(\frac{1}{t} \Delta x(t)\right)+\left(\frac{\alpha}{t^{3}}+t^{4} x^{4}(t+1)\right) x(t+1) \\
= & \frac{\beta x^{5}(t+1)}{t^{3}\left(1+x^{4}(t+1)\right)\left(1+(\Delta x(t+1))^{4}\right)}, t \geq 2,
\end{aligned}
$$

oscillates if $\alpha-\beta>1$.

In the case when $\mathbb{T}=q^{\mathbb{N}}, q>1, \sigma(s)=q s$ and $\rho(s)=\frac{s}{q}$, and one can use the fact that

$$
x_{q}^{\Delta}(t)=\frac{x(q t)-x(t)}{(q-1) t} \text { and } \int_{a}^{\infty} g(t) \Delta t=\sum_{k=0}^{\infty} \mu\left(q^{k}\right) g\left(q^{k}\right),
$$

and establish some conditions for oscillation of the $q$-difference equation $\Delta_{q}\left(\frac{1}{t} \Delta_{q} x^{\Delta}\right)+\left(\frac{\alpha}{t^{3}}+t^{4} x^{4}(q t)\right) x(q t)=\frac{\beta x^{5}(q t)}{t^{3}\left(1+x^{4}(q t)\right)\left(1+\left(\Delta_{q} x(q t)\right)^{4}\right)}, t \geq 2$, and the details are omitted due to the limited space. We note that the results that has been established by Bohner and Saker [9], can not be applied on equation (4.2). So the results in this paper improved the results in [9]. 
Acknowledgement. The author thanks Deanship of Scientific Research and the Research Centre in College of Science in King Saud University for encouragements and supporting this project.

\section{References}

[1] R. P. Agarwal, M. Bohner, D. O'Regan, and A. Peterson, Dynamic equations on time scales: a survey, Dynamic equations on time scales. J. Comput. Appl. Math. 141 (2002), no. 1-2, 1-26.

[2] R. P. Agarwal, M. Bohner, and S. H. Saker, Oscillation of second order delay dynamic equations, Can. Appl. Math. Qurt 13 (2005), no. 1, 1-17.

[3] R. P. Agarwal, D. O'Regan, and S. H. Saker, Oscillation criteria for second-order nonlinear neutral delay dynamic equations, J. Math. Anal. Appl. 300 (2004), no. 1, 203-217.

[4] E. A. Bohner, M. Bohner, and S. H. Saker, Oscillation criteria for a certain class of second order Emden-Fowler dynamic equations, Electron. Trans. Numer. Anal. 27 (2007), 1-12.

[5] E. A. Bohner and J. Hoffacker, Oscillation properties of an Emden-Fowler type equation on discrete time scales, J. Difference Eqns. Appl. 9 (2003), no. 6, 603-612.

[6] M. Bohner and A. Peterson, Dynamic Equations on Time Scales: An Introduction with Applications, Birkhäuser, Boston, 2001.

[7] _ Advances in Dynamic Equations on Time Scales, Birkhäuser, Boston, 2003.

[8] M. Bohner and S. H. Saker, Oscillation of second order nonlinear dynamic equations on time scales, Rocky Mountain J. Math. 34 (2004), no. 4, 1239-1254.

[9] - Oscillation criteria for perturbed nonlinear dynamic equations, Math. Comput. Modelling 40 (2004), no. 3-4, 249-260.

[10] L. Erbe, Oscillation criteria for second order linear equations on a time scale, Canad. Appl. Math. Quart. 9 (2001), no. 4, 345-375.

[11] L. Erbe and A. Peterson, Riccati equations on a measure chain, Dynamic systems and applications, Vol. 3 (Atlanta, GA, 1999), 193-199, Dynamic, Atlanta, GA, 2001.

[12] - Oscillation criteria for second-order matrix dynamic equations on a time scale, J. Comput. Appl. Math. 141 (2002), no. 1-2, 169-185.

[13] - Boundedness and oscillation for nonlinear dynamic equations on a time scale, Proc. Amer. Math. Soc. 132 (2004), no. 3, 735-744.

[14] L. Erbe, A. Peterson, and S. H. Saker, Oscillation criteria for second-order nonlinear dynamic equations on time scales, J. London Math. Soc. (2) 67 (2003), no. 3, 701-714.

[15] - Asymptotic behavior of solutions of a third-order nonlinear dynamic equation on time scales, J. Comput. Appl. Math. 181 (2005), no. 1, 92-102.

[16] Kamenev-type oscillation criteria for second-order linear delay dynamic equations, Dynam. Systems Appl. 15 (2006), no. 1, 65-78.

[17] G. H. Hardy, J. E. Littlewood, and G. Polya, Inequalities, 2nd Ed. Cambridge Univ. Press 1952.

[18] S. Hilger, Analysis on measure chains - a unified approach to continuous and discrete calculus, Results Math. 18 (1990), no. 1-2, 18-56.

[19] S. H. Saker, Oscillation of nonlinear dynamic equations on time scales, Appl. Math. Comput. 148 (2004), no. 1, 81-91.

[20] - Oscillation criteria of second-order half-linear dynamic equations on time scales, J. Comput. Appl. Math. 177 (2005), no. 2, 375-387.

[21] B Boundedness of solutions of second-order forced nonlinear dynamic equations, Rocky Mountain J. Math. 36 (2006), no. 6, 2027-2039.

[22] _ New oscillation criteria for second-order nonlinear dynamic equations on time scales, Nonlinear Funct. Anal. Appl. 11 (2006), no. 3, 351-370. 
[23] - Oscillation of second-order nonlinear neutral delay dynamic equations on time scales, J. Comput. Appl. Math. 187 (2006), no. 2, 123-141.

Department of Mathematics

College of SCience

King SAUd University

RIYADH 11451, SAUDi ARABia

AND

Department of Mathematics

FACULTY OF SCIENCE

MANSOURA UNIVERSITY

MANSOURA 35516, EGYPT

E-mail address: shsaker@mans.edu.eg 